\title{
Approximate Newton Methods for Nonsmooth Equations ${ }^{1}$
}

\author{
H. XU ${ }^{2}$ AND $X . W \cdot$ ChANG $^{3}$
}

Communicated by D. Q. Mayne

\begin{abstract}
We develop general approximate Newton methods for solving Lipschitz continuous equations by replacing the iteration matrix with a consistently approximated Jacobian, thereby reducing the computation in the generalized Newton method. Locally superlinear convergence results are presented under moderate assumptions. To construct a consistently approximated Jacobian, we introduce two main methods: the classic difference approximation method and the $\epsilon$-generalized Jacobian method. The former can be applied to problems with specific structures, while the latter is expected to work well for general problems. Numerical tests show that the two methods are efficient. Finally, a normreducing technique for the global convergence of the generalized Newton method is briefly discussed.
\end{abstract}

Key Words. Lipschitz continuous equations, semismooth equations, consistently approximated Jacobians, difference approximations, $\epsilon$-generalized approximate Jacobians.

\section{Introduction}

We study the following system of nonsmooth equations:

$$
F(x)=0 \text {, }
$$

where $F: R^{n} \rightarrow R^{n}$ is locally Lipschitz continuous.

\footnotetext{
'The authors are grateful to the referee and D. Q. Mayne for their insightful comments and constructive suggestions. Thanks go to L. Qi and M. Kojima for providing some helpful references and to $\mathbf{S}$. Leyffer for many helpful discussions.

${ }^{2}$ Lecturer, Department of Mathematics, Ningbo University, Ningbo, Zhejiang Province, P.R. China; Visiting Scholar, Department of Mathematics and Computer Sciences, University of Dundee, Dundee, Scotland.

${ }^{3}$ Graduate Student, School of Computer Science, McGill University, Montréal, Québec, Canada.
} 
Since the introduction of B-derivatives by Robinson (Ref. 1), efforts have been made to generalize the Newton method and quasi-Newton methods to the nondifferentiable case. Among them are Refs. 2-10, most of which are intended to solve some specific nondifferentiable equations such as those transformed from nonlinear complementarity problems and mathematical programming problems. Kojima and Shindo (Ref. 11) first discussed a Newton-like method for systems of piecewise continuously differentiable equations. Ip and Kyparisis (Ref. 12) extended the classic quasi-Newton methods, especially the Broyden method, to B-differentiable equations. Locally superlinear convergence results were obtained under the condition that $F(x)$ has Gateaux derivatives at a solution point of (1). However, the extension seems to be limited, since a bound on the deterioration of the updating matrix cannot be maintained if $F$ is not differentiable at a solution point.

Qi and Sun (Ref. 13) proposed a generalized Newton method for (1). They employed the following iteration:

$$
x_{k+1}=x_{k}-V_{k}^{-1} F\left(x_{k}\right), \quad V_{k} \in \partial F\left(x_{k}\right),
$$

where $\partial F\left(x_{k}\right)$ is the generalized Jacobian of $F$ at $x_{k}$, defined by Clarke (Ref. 14), and $V_{k}$ is arbitrarily taken from $\partial F\left(x_{k}\right)$. The iterates produced by (2) were proved to be locally superlinearly convergent under mild conditions. However, difficulties may occur when (2) is applied to solve real-life problems. The obvious one is the calculation of $V_{k}$ when $F$ is not differentiable at $x_{k}$. A simple idea that we are familiar with is to replace $V_{k}$ with the Jacobian of $F$ at a point near $x_{k}$, but the computation of a Jacobian is also a formidable task, even in the smooth case. Another difficulty is that the iteration (2) may not work well if there is no significant reduction of $\|F(x)\|$ when the initial guess is far from a solution point.

Pang and Qi (Ref. 15) developed a new kind of Gauss-Newton method for solving a certain class of nonsmooth equations. They also extended the classical superlinear convergence results of Dennis and Moré for smooth equations and those of Ip and Kyparisis for B-differentiable equations. There are also other new surveys on the topic; see Refs. 16-18, for instance.

In this paper, we develop general approximate Newton methods for (1) in order to avoid the complicated computation of $V_{k}$ in (2). In Section 2, we establish a general approximate Newton iterative scheme by introducing the concept of consistently approximated Jacobian (CAJ in brief); local convergence results are presented under some mild conditions. In Section 3, we propose two practical methods: the classic difference approximation method and the $\epsilon$-generalized Jacobian method. The former can be applied to a few problems with specific structures, while the latter is expected to work well for more general problems. In Section 4, we introduce briefly a 
norm reducing technique to force the generalized Newton algorithms to converge when the initial point is far from a solution point of (1). Finally, we give numerical experiments in Section 5.

\section{General Approximate Newton Iteration}

2.1. Notation. Throughout this paper, we use the following notation. $R^{n}$ denotes the vector space of $n$-tuples with 2 -norm $\|\cdot\|$, and $L\left(R^{n}\right)$ the matrix space of $n \times n$ real matrices with the induced norm $\|\cdot\| . S(x, \delta)$ denotes the open ball in $R^{n}$ with center $x$ and radius $\delta$, and $B$ is an open unit ball in $L\left(R^{n}\right)$. The closure of a set $D$ is specified by $\bar{D}$. If $\alpha(h)$ is a vector-valued function (in this context, we simply call it a function for brevity) or a matrix function of $h \in R^{n}$, we use $\alpha(h)=o(\|h\|)$ to denote the case where

$$
\|\alpha(h)\| /\|h\| \rightarrow 0, \quad \text { as }\|h\| \rightarrow 0 ;
$$

and we use $\alpha(h)=O(\|h\|)$ if there exist constants $C_{1}, C_{2}>0$ such that

$$
C_{1}\|h\| \leq \alpha(h) \leq C_{2}\|h\|, \quad \text { as }\|h\| \rightarrow 0 .
$$

The set of positive integers $[1, \ldots, n]$ is denoted by $\bar{n}$. The set of points of $R^{n}$ at which $F$ is differentiable is denoted by $D_{F}$.

2.2. Basics. We assume throughout this paper that $F$ is locally Lipschitz continuous in $R^{n}$ in the sense that, for every $x$, there exist $L>0$ and $\delta>0$, such that

$$
\|F(y)-F(z)\| \leq L\|y-z\|,
$$

for all $y, z \in S(x, \delta)$. Here, $L$ is called the Lipschitz constant of $F$ at $x$.

According to the Rademacher theorem, $F$ is differentiable almost everywhere in $R^{n}$. The generalized Jacobian of $F$ at $x$ is defined by Clarke in Ref. 14 as follows:

$$
\partial F(x)=\operatorname{conv}\left[\lim _{x_{1} \rightarrow x_{,}, x_{i} \in D_{F}} \nabla F\left(x_{i}\right)\right] .
$$

Here and later on, $\nabla F(x)$ denotes the Jacobian of $F$ at $x \in D_{F}$; conv denotes the convex hull.

Proposition 2.1. See Proposition 2.6.2., Ref. 14. $\partial F(x)$ is compact and upper semicontinuous in the sense that, for every $\epsilon>0$, there exists $\delta>0$ such that

$$
\partial F(y) \subset \partial F(x)+\epsilon B, \quad \forall y \in S(x, \delta) .
$$


For the sake of convenience in the subsequent discussion, we summarize some preliminary concepts and results given by Qi and Sun in Ref. 13. $F$ is said to be semismooth at $x$ if, for every $h \in R^{n}$, the following limit exists:

$$
\lim _{V \in \partial F\left(x+t h^{\prime}\right), h^{\prime} \rightarrow h, t \rightarrow 0} V h^{\prime} .
$$

Lemma 2.1. Suppose that $F$ is semismooth at $x$. Then, for every $h \in R^{n}$,

$$
\lim _{h^{\prime} \rightarrow h, t \rightarrow 0}\left[F\left(x+t h^{\prime}\right)-F(x)\right] / t=\lim _{V \in \partial F\left(x+t h^{\prime}\right), h^{\prime} \rightarrow h, t \rightarrow 0} V h^{\prime}=F^{\prime}(x, h) .
$$

In particular, when $h^{\prime} \equiv h$,

$$
F^{\prime}(x, h)=\lim _{V \in \partial F(x+t h), t \rightarrow 0} V h \in \partial F(x) h
$$

and

$$
F(x+h)-F(x)-F^{\prime}(x, h)=o(\|h\|) .
$$

Proof. It follows directly from Propositions 2.1 and 2.17 of Ref. 13 and the definition of semismoothness.

If there exists $0 \leq p<1$ such that, for any $V \in \partial F(x+h), h \rightarrow 0$,

$$
V h-F^{\prime}(x, h)=O\left(\|h\|^{1+p}\right),
$$

then $F$ is said to be $p$-order semismooth at $x$. In Ref. 13, Qi and Sun proved that, if $F$ is $P$-order semismooth at $x$, then

$$
F(x+h)-F(x)-F^{\prime}(x, h)=O\left(\|h\|^{1+p}\right) .
$$

Lemma 2.2. See Lemma 2.2, Ref. 13. Suppose that $F^{\prime}(x, h)$ exists at $x$ for any $h$. Then for every $h$, there exists $V \in \partial F(x)$ such that

$$
F^{\prime}(x, h)=V h \text {. }
$$

Lemma 2.3. See Theorem 2.3, Ref. 13. The following statements are equivalent:

(a) $F$ is semismooth at $x$;

(b) for any $V \in \partial F(x+h), h \rightarrow 0, V h-F^{\prime}(x, h)=o(\|h\|)$;

(c) $\lim _{h \rightarrow 0}\left[F^{\prime}(x+h, h)-F^{\prime}(x, h)\right] /\|h\|=0$.

$\partial F(x)$ is said to be nonsingular if all $V \in \partial F(x)$ are nonsingular.

Lemma 2.4. See Proposition 3.1, Ref. 13. Suppose that $\partial F(x)$ is nonsingular. Then, there exist $\delta>0, C_{1}>0$, such that, for each $y \in S(x, \delta), \partial F(y)$ 
is nonsingular and

$$
\left\|V^{-1}\right\| \leq C_{1}, \quad \forall V \in \partial F(y), y \in S(x, \delta) .
$$

2.3. Approximate Newton Method. We now introduce an approximate Newton iteration. Let $x_{k}$ be the current point, and let $x_{k+1}$ be the next point. Then, $x_{k+1}$ is computed by

$$
x_{k+1}=x_{k}-J\left(x_{k}, s_{k}\right)^{-1} F\left(x_{k}\right),
$$

where $J\left(x_{k}, s_{k}\right) \in L\left(R^{n}\right)$ is an approximation of some $V_{k} \in \partial F\left(x_{k}\right)$ and $s_{k}$ is an $l$-dimensional parameter vector.

The iteration (4) is a generalization of approximate Newton methods in the smooth case, which were discussed extensively by Ortega and Rheinboldt in Ref. 19. The main difficulty here is the construction of $J(x, s)$. For the sake of convenience, we present a strict theoretical definition of $J(x, s)$.

Let $N(x, \delta)$ denote the closed ball with center $x$ and radius $\delta$ in $R^{\prime}$ in order to distinguish it from $S(x, \delta)$.

Definition 2.1. Let $J: D \times D_{l} \subset R^{n} \times R^{l} \rightarrow L\left(R^{n}\right)$. If $0 \in R^{\prime}$ is a limiting point of $D_{l}$, and if

$$
\lim _{s \rightarrow 0} \operatorname{dist}[J(x, s) ; \partial F(x)]=0,
$$

for all $x \in D$, then $J(x, s)$ is called a consistently approximated Jacobian of $F$ in $D$. We call it CAJ for brevity. Here,

$$
\operatorname{dist}[J(x, s) ; \partial F(x)] \equiv \min _{V \in \partial F(x)}\|J(x, s)-V\| .
$$

Lemma 2.5. Let $x^{*} \in R^{n}$. Suppose that $F$ is semismooth at $x^{*}$ and that $J(x, s)$ is a CAJ of $F(x)$ in a neighborhood of $x^{*}$. Then,

$$
\left\|F^{\prime}\left(x^{*}+h, h\right)-J\left(x^{*}+h, s\right) h\right\| \leq w(h, s)\|h\|,
$$

where

$$
\lim _{h \rightarrow 0, s \rightarrow 0} w(h, s)=0
$$

Proof. Let $\delta>0$ be sufficiently small so that $J(x, s)$ is a CAJ of $F$ in $S\left(x^{*}, \delta\right)$. By Definition 2.1,

$$
\lim _{s \rightarrow 0} \operatorname{dist}\left[J\left(x^{*}+h, s\right) ; \partial F\left(x^{*}+h\right)\right]=0,
$$


uniformly with respect to $\|h\| \leq \delta$. Note that $\partial F\left(x^{*}+h\right)$ is closed. Then for each $s$, there exists a $V\left(x^{*}+h, s\right) \in \partial F\left(x^{*}+h\right)$ such that

$$
\begin{aligned}
& \left\|V\left(x^{*}+h, s\right)-J\left(x^{*}+h, s\right)\right\| \\
& =\operatorname{dist}\left[J\left(x^{*}+h, s\right) ; \partial F\left(x^{*}+h\right)\right] \rightarrow 0,
\end{aligned}
$$

uniformly with respect to $\|h\| \leq \delta$, as $s \rightarrow 0$. Since $F$ is semismooth at $x^{*}$, from Lemma 2.3 (b),

$$
V\left(x^{*}+h, s\right) h-F^{\prime}\left(x^{*}, h\right)=o(\|h\|),
$$

uniformly with respect to $s$. Using Lemma 2.3 (c), we get

$$
F^{\prime}\left(x^{*}+h, h\right)-F^{\prime}\left(x^{*}, h\right)=o(\|h\|) \text {. }
$$

Write

$$
\begin{aligned}
w(h ; s) \equiv & \left\|V\left(x^{*}+h\right)-J\left(x^{*}+h, s\right)\right\| \\
& +\left[\left\|F^{\prime}\left(x^{*}+h, h\right)-F^{\prime}\left(x^{*}, h\right)\right\|\right. \\
& \left.+\left\|V\left(x^{*}+h, s\right) h-F^{\prime}\left(x^{*}, h\right)\right\|\right] /\|h\| .
\end{aligned}
$$

Then, we see that (8) holds from (9)-(11) and

$$
\begin{aligned}
& \left\|F^{\prime}\left(x^{*}+h, h\right)-J\left(x^{*}+h, s\right) h\right\| \\
& \leq\left\|F^{\prime}\left(x^{*}+h, h\right)-F^{\prime}\left(x^{*}, h\right)\right\|+\left\|V\left(x^{*}+h, s\right) h-F^{\prime}\left(x^{*}, h\right)\right\| \\
& +\left\|V\left(x^{*}+h, s\right)-J\left(x^{*}+h, s\right)\right\|\|h\| \\
& =w(h, s)\|h\| .
\end{aligned}
$$

The proof is complete.

Now, we can state our main results.

Theorem 2.1. Let $x^{*} \in D$ be a solution of (1). Suppose that $F$ is semismooth at $x^{*}$ and that $\partial F\left(x^{*}\right)$ is nonsingular. Let $J: D \times D_{l} \subset R^{n} \times R^{\prime} \rightarrow L\left(R^{n}\right)$ be a CAJ of $F$ in $D$, and assume that $0 \in D_{l}$. Then, there exist $\delta>0$ and $\gamma>0$ such that $J(x, s)$ is nonsingular for $x \in S\left(x^{*}, \delta\right), s \in N(0, \gamma)$. Furthermore, the function

$$
G(x, s) \equiv x-J(x, s)^{-1} F(x)
$$

is contractive in the sense that

$$
\begin{aligned}
& \left\|x^{*}-G(x, s)\right\| \leq u(x, s)\left\|x-x^{*}\right\|, \\
& \forall x \in D \cap S\left(x^{*}, \delta\right), s \in D, \cap N(0, \gamma),
\end{aligned}
$$


where

$$
\lim _{x \rightarrow x^{*}, s \rightarrow 0} u(x, s)=0
$$

Proof. First, we show that there exist $\delta>0$ and $\gamma>0$, such that $J(x, s)$ is nonsingular for any $x \in D \cap S\left(x^{*}, \delta\right)$ and any $s \in D, \cap N(0, \gamma)$. It follows from Definition 2.1 that, for each $\epsilon>0$, there exists a constant $\gamma>0$ such that

$$
\operatorname{dist}[J(x, s) ; \partial F(x)] \leq \epsilon, \quad \forall x \in D, s \in D_{l} \cap N(0, \gamma) .
$$

Since $\partial F\left(x^{*}\right)$ is nonsingular, by Lemma 2.4 , there exist a constant $C_{1}>0$ and $\delta>0$, such that $\partial F(x)$ is nonsingular and

$$
\left\|V(x)^{-1}\right\| \leq C_{1}, \quad \forall x \in D \cap S\left(x^{*}, \delta\right) .
$$

Let

$$
\hat{V}(x) \equiv \underset{V \in i F(x)}{\arg \min }\|J(x, s)-V(x)\| .
$$

Then, from (15) and (6), we have

$$
\|J(x, s)-\hat{V}(x)\| \leq \epsilon .
$$

Suppose without loss of generality that $\epsilon C_{1}<1$. Then, by the Banach perturbation theorem and $(16), J(x, s)$ is nonsingular and satisfies

$$
\begin{aligned}
\left\|J(x, s)^{-1}\right\| & \leq\left\|\hat{V}(x)^{-1}\right\| /\left(1-\left\|\hat{V}(x)^{-1}\right\|\|J(x, s)-\hat{V}(x)\|\right) \\
& \leq C_{1} /\left(1-\epsilon C_{1}\right) .
\end{aligned}
$$

Now, we prove (13). In fact,

$$
\begin{aligned}
& \left\|x^{*}-G(x, s)\right\| \\
& =\left\|J(x, s)^{-1}\left[F(x)-J(x, s)\left(x-x^{*}\right)\right]\right\| \\
& \leq\left\|J(x, s)^{-1}\right\|\left[\left\|F(x)-F\left(x^{*}\right)-F^{\prime}\left(x^{*}, x-x^{*}\right)\right\|\right. \\
& \quad+\left\|F^{\prime}\left(x, x-x^{*}\right)-F^{\prime}\left(x^{*}, x-x^{*}\right)\right\| \\
& \left.\quad+\left\|F^{\prime}\left(x, x-x^{*}\right)-J(x, s)\left(x-x^{*}\right)\right\|\right] \\
& \leq C_{1} /\left(1-\epsilon C_{1}\right)\left[o\left(\left\|x-x^{*}\right\|\right)+o\left(\left\|x-x^{*}\right\|\right)+w\left(x-x^{*}, s\right)\left\|x-x^{*}\right\|\right] \\
& \equiv u(x, s) O\left(\left\|x-x^{*}\right\|\right), \text { say. }
\end{aligned}
$$

The last inequality is due to Lemma 2.3 , Lemma 2.5 , (3), and (17). Clearly, (14) holds. The proof is complete. 
Corollary 2.1. Suppose that the assumptions in Theorem 2.1 are satisfied. Let $G(x, s)$ be defined by (12), and let $g: R^{n} \rightarrow R^{s}$ be a continuous function with $g\left(x^{*}\right)=0$. If $s=g(x)$, then there exists $\delta>0$ such that $G(x, g(x))$ is contractive in $S\left(x^{*}, \delta\right)$. Thereby, the sequence produced by (4) converges to $x^{*}$ superlinearly for a sufficiently good starting point.

Proof. From (14), it follows that, for any given $\epsilon>0$, there exist $\delta_{1}>0$ and $\sigma>0$ such that

$$
u(x, s) \leq \epsilon, \quad \forall x \in S\left(x^{*}, \delta_{1}\right),\|s\| \leq \sigma .
$$

Since $s=g(x)$ and $g$ is continuous at $x^{*}$ with $g\left(x^{*}\right)=0$, there exists $\delta<\delta_{1}$ such that

$$
\|\boldsymbol{s}\|=\|g(x)\| \leq \sigma, \quad \forall x \in S\left(x^{*}, \delta\right) ;
$$

thereby,

$$
u(x, g(x)) \leq \epsilon, \quad \forall x \in S\left(x^{*}, \delta\right) .
$$

The conclusion is obvious as $\epsilon$ can be arbitrarily small.

\section{Practical Approximation Methods}

In Section 2, we established a unified framework of superlinearly convergent approximate Newton methods based on CAJ. The results may be regarded as theoretical, since there are no practical methods presented for the construction of a CAJ. In this section, we discuss how to construct a CAJ practically. Obviously, the existence of a CAJ depends on the local property of $F(x)$. It seems unlikely to provide a unified approach for general problems. In what follows, we propose two methods: the finite difference approximation method and the $\epsilon$-generalized Jacobian approximation method.

3.1. Finite Difference Approximation. We first introduce some useful notation. If $a_{j}, j \in \bar{n}$, are $n$ vectors of $R^{n}$, we will denote by $\left[a_{1}, \ldots, a_{n}\right]$ the matrix whose $j$ th column is $a_{j}$. Similarly if $A_{j}, j \in \bar{n}$, are subsets of $R^{n}$, then $\left[A_{1}, \ldots, A_{n}\right]$ will be $\left[\left[a_{1}, \ldots, a_{n}\right]: a_{j} \in A_{j}\right.$, for all $\left.j \in \bar{n}\right]$.

Now, we suppose that $F$ is semismooth in $R^{n}$. By Lemma 2.1, for each $x \in R^{n}$,

$$
F^{\prime}\left(x, e_{j}\right) \in \partial F(x) e_{j}, \quad j \in \bar{n},
$$


where $e_{j}$ is the $j$ th unit vector. On the other hand,

$$
\partial F(x) e_{j} e_{j}^{T}=\left[0, \ldots, \partial_{j} F, \ldots, 0\right], \quad j \in \bar{n},
$$

where

$$
\partial_{j} F=\operatorname{conv}\left[\lim _{x_{i} \in D_{F}, x_{i} \rightarrow x} \nabla_{j} F\left(x_{i}\right)\right]
$$

and $\partial_{j} F$ denotes the derivatives of $F$ with respect to the $j$ th component of the variable $x$ at a point $x_{i} \in D_{F}$. Hence,

$$
F^{\prime}\left(x, e_{j}\right) e_{j}^{T} \in\left[0, \ldots, \partial_{j} F, \ldots, 0\right], \quad j \in \bar{n} ;
$$

consequently,

$$
\sum_{j \in \bar{n}} F^{\prime}\left(x, e_{j}\right) e_{j}^{T} \in\left[\partial_{1} F, \ldots, \partial_{n} F\right] .
$$

In general,

$$
\left[\partial_{1} F, \ldots, \partial_{n} F\right] \neq \partial F
$$

but equality holds at least in two particular cases: first, when all functions $f_{j}$, except possibly one (say $f_{1}$ ), are strictly differentiable at $x$, in such a case,

$$
\partial F=\left[\partial f_{1}, \nabla f_{2}, \ldots, \nabla f_{n}\right]^{r} ;
$$

another equality case is when the functions are nondifferentiable but only with respect to nonrelated variables.

Example 3.1. Let $F: R^{2} \rightarrow R^{2}$ be defined by

$$
F(x)=\left(\left|x_{1}\right|+x_{2}, x_{1}+\left|x_{2}\right|\right)^{T} .
$$

Then,

$$
\partial F=\left\{\left[\begin{array}{ll}
\alpha & 1 \\
1 & \beta
\end{array}\right], \alpha \in[-1,1], \beta \in[-1,1]\right\}=\left[\partial_{1} F, \partial_{2} F\right] .
$$

So far, we are able to reach the following conclusions.

Lemma 3.1. Suppose that $F$ is a function whose components are either nondifferentiable only with respect to nonrelated variables or strictly differentiable, except for one, and $F$ is semismooth in $R^{n}$. Then,

$$
J(x, s) \equiv \sum_{j \in \bar{n}}\left[\left[F\left(x+s e_{j}\right)-F(x)\right] / s\right] e_{j}^{T} \in \partial F(x)+o(s) .
$$


Proof. Since $F(x)$ is semismooth, by Lemma 2.1,

$$
\left[F\left(x+s e_{j}\right)-F(x)\right] / s-F^{\prime}\left(x, e_{j}\right)=o(s), \quad j \in \bar{n} .
$$

Hence,

$$
\begin{aligned}
J(x, s) & =\left[\sum_{j \in \bar{n}}\left[F\left(x+s e_{j}\right)-F(x)\right] / s\right] e_{j}^{T} \\
& =\sum_{j \in \bar{n}} F^{\prime}\left(x, e_{j}\right) e_{j}^{T}+o(s) \in\left[\partial_{1} F, \ldots, \partial_{n} F\right]+o(s) .
\end{aligned}
$$

By assumption, we get

$$
\left[\partial_{1} F, \ldots, \partial_{n} F\right]=\partial F(x) .
$$

This completes the proof.

Theorem 3.1. Let $x^{*}$ be a solution of (1). Assume that $F$ is semismooth in a neighborhood of $x^{*}$ and that $\partial F\left(x^{*}\right)$ is nonsingular. Suppose that the assumptions of Lemma 3.1 are satisfied and that $J(x, s)$ is defined by (19), which holds uniformly with respect to $x$ in a neighborhood of $x^{*}$. Then, for $s=O(\|F(x)\|)$, there exists $\delta>0$ such that, if the starting point $x_{0} \in S\left(x^{*}, \delta\right)$, the sequence $\left\{x_{k}\right\}$ generated by (4) is well defined and converges to $x^{*}$ superlinearly.

Proof. It follows from Lemma 3.1 that $J(x, s)$ is a CAJ of $F$ in some neighborhood of $x^{*}$ under the assumption that (18) holds uniformly with respect to $x$ in a neighborhood of $x^{*}$. The rest follows directly from Corollary 2.1.

Remark 3.1. Theorem 3.1 is a nonsmooth version of the classic difference approximate Newton method. It seems that the nonsmooth finite approximation only fits a small class of problems. Therefore, we are led to develop other techniques which are likely to deal with more general problems.

3.2. E-Generalized Jacobian Approximation. In nonsmooth optimization, when the steepest subgradient method failed to solve the Wolfe counterexample, people realized that the subdifferential of a nonsmooth function contains too little information for finding a convergent search direction. A larger set, called $\epsilon$-subdifferential, was then introduced and the existing difficulties were consequently overcome. In this context, using the same method as for defining the $\epsilon$-subdifferential, we try to define an $\epsilon$-generalized Jacobian for a vector-valued function. However, rather than in light of 
convergence considerations, the definition here is intended to provide more practical substitutes of the generalized Jacobians, which are usually difficult to compute.

Definition 3.1. Let $F(x)$ be locally Lipschitz continuous. Then, we say that $\partial_{\epsilon} F(x)$ is the $\epsilon$-generalized Jacobian of $F$ at $x$ if

$$
\partial_{\epsilon} F(x)=\operatorname{conv}\left[\partial F\left(x^{\prime}\right): x^{\prime} \in \overline{S(x ; \epsilon)}\right] .
$$

The $\epsilon$-generalized Jacobian has many interesting properties.

Proposition 3.1. Let $\partial_{\epsilon} F(x)$ be defined by (19). Then:

(a) for every $x \in R^{n}, \partial_{0} F(x)=\partial F(x)$;

(b) for every $x \in R^{n}$, if $\epsilon<\epsilon^{\prime}$, then $\partial_{\epsilon} F(x) \subset \partial_{\epsilon} F(x)$;

(c) for any $\epsilon \geq 0, \partial_{\epsilon} F(x)$ is convex and bounded on a bounded set;

(d) for every $\mu>0$, there exists $\delta>0$ such that

$$
\partial_{\epsilon} F(y) \subset \partial F(x)+\mu B, \quad \forall y \in S(x, \delta), \epsilon<\delta .
$$

Proof. We only prove (d). By Proposition 2.1, for every $\mu>0$, there exists $\delta>0$ such that

$$
\partial F\left(x^{\prime}\right) \subset \partial F(x)+\mu B, \quad \forall x^{\prime} \in S(x, \delta) .
$$

From Definition 3.2, we have, for $\epsilon<\delta / 2, y \in S(x, \delta / 2)$,

$$
\begin{aligned}
\partial_{\epsilon} F(y) & =\operatorname{conv}\left[\partial F\left(x^{\prime}\right): x^{\prime} \in \overline{S(y, \epsilon)}\right] \\
& \subset \operatorname{conv}\left[\partial F\left(x^{\prime}\right): x^{\prime} \in S(x, \delta)\right] \subset \partial F(x)+\mu B .
\end{aligned}
$$

Proposition 3.2. For each $V_{\epsilon} \in \partial_{\epsilon} F(x)$,

$$
\lim _{\epsilon \rightarrow 0} V_{\epsilon} \in \partial F(x)
$$

Proof. Note that, for $V_{\epsilon} \in \partial_{\epsilon} F(x), \epsilon_{0}>0,\left\{V_{\epsilon}\right\}$ is bounded for $\epsilon \in\left[0, \epsilon_{0}\right]$. Assume that there is a subsequence $\left\{V_{\epsilon_{j}}\right\}$, with $V_{\epsilon_{j}} \rightarrow V^{\prime} \notin \partial F(x)$ as $\epsilon_{j} \rightarrow 0$, and seek a contradiction. Since

$$
\partial F(x)=\bigcap_{0 \leq \epsilon \leq \epsilon_{0}} \partial_{\epsilon} F(x),
$$

we have

$$
V^{\prime} \notin \bigcap_{0 \leq \epsilon \leq \epsilon_{0}} \partial_{\epsilon} F(x)
$$


or equivalently,

$$
V^{\prime} \in\left(\bigcap_{0 \leq \epsilon \leq \epsilon_{0}} \partial_{\epsilon} F(x)\right)^{c}=\bigcup_{0 \leq \epsilon \leq \epsilon_{0}}\left(\partial_{\xi} F(x)\right)^{c},
$$

where $A^{c}$ denotes the complementary set of $A$. Thus, there exists $0 \leq \epsilon^{\prime} \leq \epsilon_{0}$ such that

$$
V^{\prime} \in\left(\partial_{\epsilon^{\prime}} F(x)\right)^{c} .
$$

On the other hand, let $j$ be so large that $\epsilon_{j}<\epsilon^{\prime}$. Then, by Proposition 3.1 (b),

$$
V_{\epsilon_{l}} \in \partial_{\epsilon_{i}} F(x) \subset \partial_{\epsilon^{\prime}} F(x) .
$$

Since $\partial_{\epsilon^{\prime}} F(x)$ is compact, we have

$$
V^{\prime} \in \partial_{\epsilon^{\prime}} F(x),
$$

which leads to a contradiction to (21).

In general, it is very restrictive if we require (20) to hold uniformly with respect to $x$ in some open set $D$. Therefore, $V_{\epsilon}$ is not necessarily a CAJ in general cases. This leads us to consider a weaker condition for the convergence of the approximate Newton methods.

Lemma 3.2. Suppose that $F$ is semismooth at $x^{*}$ and that $E: R_{+} \rightarrow R_{+}$ is a real function with $E(0)=0$ and $\lim _{t \rightarrow 0} E(t) / t=0$. Then, for every $U \in \partial_{E\left(\left\|x-x^{*}\right\|\right)} F(x)$, there exists $V \in \partial F(x)$ such that

$$
\lim _{x \rightarrow x^{*}}(U-V)\left(x-x^{*}\right) /\left\|x-x^{*}\right\|=0 .
$$

Proof. Let

$$
t \equiv\left\|x-x^{*}\right\|, \quad h \equiv\left(x-x^{*}\right) /\left\|x-x^{*}\right\| .
$$

Then, for each $U \in \partial_{E\left(\left\|x-x^{*}\right\|\right)} F(x)$, we have by Definition 3.2 and the Carathedory theorem that

$$
U=\sum_{i=1}^{l(x)} \alpha_{i}(x) U_{i}(x)
$$

Here, $l(x)$ is an upper-bounded positive number; $\alpha_{i}(x), i=1, \ldots, l(x)$, are nonnegative scalars with $\sum_{i=1}^{\prime(x)} \alpha_{i}(x)=1$; and

$$
U_{i}(x) \in \partial F\left(x^{*}+t h+\theta_{i}(x) E(t) s_{i}(x)\right),
$$


with $s_{i}(x) \in R^{n},\left\|s_{i}(x)\right\|=1$, and $\theta_{i}(x) \in[0,1]$, for $i=1, \ldots, l(x)$. Let

$$
h_{i}(t) \equiv h+\theta_{i}(x) E(t) s_{i}(x) / t .
$$

Suppose without loss of generality that $h \rightarrow \hat{h}$ as $t \rightarrow 0$. Since $F$ is semismooth at $x^{*}$, it follows from Lemma 2.1 that, for every $V \in \partial F(x)$,

$$
\lim _{t \rightarrow 0} U_{i}(x) h_{i}(t)=\lim _{t \rightarrow 0} U_{i}(x) h=\lim _{i \rightarrow 0} V h=F^{\prime}\left(x^{*}, \hat{h}\right),
$$

which implies (22).

Remark 3.2. The conclusion of Lemma 3.2 still holds when $E(t)$ is replaced by $k E(t)$ for some positive constant $k$.

Theorem 3.2. Let $x^{*}$ be a solution point of (1). Suppose that $F$ is semismooth at $x^{*}$ and that $\partial F\left(x^{*}\right)$ is nonsingular. Let $E: R_{+} \rightarrow R_{+}$be a real function with $E(0)=0$ and $\lim _{t \rightarrow 0} E(t) / t=0$. Then, the iteration

$$
x_{k+1}=x_{k}-U_{k}^{-1} F\left(x_{k}\right), \quad U_{k} \in \partial_{E\left(\left\|x_{k}-x^{*}\right\|\right)} F\left(x_{k}\right),
$$

is well defined and superlinearly convergent to $x^{*}$ in a neighborhood of $x^{*}$.

Proof. Since $\partial F\left(x^{*}\right)$ is nonsingular, by Proposition $3.1(\mathrm{~d})$, there exists $\delta>0$ such that $\partial_{\epsilon} F(x)$ is also nonsingular and $\left\|U^{-1}\right\|$ is bounded for every $U \in \partial_{\epsilon} F(x)$, when $\epsilon<\delta, x \in S\left(x^{*}, \delta\right)$. Hence,

$$
\begin{aligned}
\left\|x_{k+1}-x^{*}\right\| \leq & \left\|U_{k}^{-1}\right\|\left[\left\|F\left(x_{k}\right)-F\left(x^{*}\right)-F^{\prime}\left(x^{*}, x_{k}-x^{*}\right)\right\|\right. \\
& +\left\|F^{\prime}\left(x_{k}, x-x^{*}\right)-F^{\prime}\left(x^{*}, x-x^{*}\right)\right\| \\
& \left.+\left\|F^{\prime}\left(x_{k}, x-x^{*}\right)-V_{k}\left(x_{k}-x^{*}\right)\right\|+\left\|\left(U_{k}-V_{k}\right)\left(x_{k}-x^{*}\right)\right\|\right] \\
= & o\left(\left\|x_{k}-x^{*}\right\|\right) .
\end{aligned}
$$

The last equality follows from Lemma 2.1, Lemma 2.3, and Lemma 3.2. The proof is complete.

The methods for the construction of $\partial_{\epsilon} F(x)$ vary from problem to problem. For some problems, a detailed knowledge of $\partial_{\epsilon} F(x)$ is needed; for some others, maybe one element of $\partial_{\epsilon} F(x)$ is enough. In what follows, we present an integral method which can be regarded as a generalization of the results of Ref. 20.

Let $\|\cdot\|_{\infty}$ denote the infinity norm of vectors of $R^{n}$; set

$$
P_{\epsilon}(x) \equiv\left[y \in R^{n}:\|y-x\|_{\infty} \leq \epsilon\right] .
$$


Define

$$
F_{\epsilon}(x) \equiv\left(f_{\epsilon}^{1}(x), \ldots, f_{\epsilon}^{n}(x)\right)^{T},
$$

where

$$
f_{\epsilon}^{i}(x) \equiv \sigma(\epsilon) \int_{P_{\epsilon}(x)} f_{i}(y) d y, \quad \sigma(\epsilon)=1 /(2 \epsilon)^{n} .
$$

Then, we have the following proposition.

Proposition 3.3. $F_{\epsilon}(x)$ is continuously differentiable with respect to $x$ and

$\nabla_{j} f_{\epsilon}^{i}(x)=\left[1 /(2 \epsilon)^{n}\right]\left[\int_{D_{\epsilon^{\prime}+(x)}} f_{i}(y) d y-\int_{D_{\epsilon-(}^{\prime}(x)} f_{i}(y) d y\right], \quad i, j=1, \ldots, n$,

where

$$
\begin{aligned}
D_{\epsilon+}^{j}(x) & =\left[y \in R^{n}:\|y-x\|_{\infty}=\epsilon,(y)_{j}=(x)_{j}+\epsilon\right] \\
& =\left[x+s:(s)_{j}=\epsilon,\left|(s)_{i}\right| \leq \epsilon, i \neq j, 1 \leq i \leq n\right], \\
D_{\epsilon-}^{j}(x) & =\left[y \in R^{n}:\|y-x\|_{\infty}=\epsilon,(y)_{j}=(x)_{j}-\epsilon\right] \\
& =\left[x-s:(s)_{j}=\epsilon,\left|(s)_{j}\right| \leq \epsilon, i \neq j, 1 \leq i \leq n\right],
\end{aligned}
$$

and where $(x)_{j}$ denotes the $j$ th component of vector $x$.

Proof. The proof can be obtained by a simple calculation.

Lemma 3.3. Let $F_{\epsilon}(x)$ be defined by (24). Then, for every $h \in R^{n}$ with $\|h\|=1$,

$$
\nabla F_{\epsilon}(x) h \in \partial_{(\sqrt{n}+1) \epsilon} F(x) h .
$$

Proof. Let $h \in R^{n}$ with $\|h\|=1$. Since $F_{\epsilon}(x)$ is differentiable,

$$
\begin{aligned}
\nabla F_{\epsilon}(x) h & =\lim _{t \rightarrow 0}\left[F_{\epsilon}(x+t h)-F_{\epsilon}(x)\right] / t \\
& =\lim _{t \rightarrow 0} \sigma(\epsilon) \int_{P_{\epsilon}(0)}\left[\left(F_{\epsilon}(x+y+t h)-F_{\epsilon}(x+y)\right) / t\right] d y \\
& =\lim _{t \rightarrow 0} \sigma(\epsilon) \int_{P_{\epsilon}(0)} \int_{0}^{1} \nabla F(x+s+t \theta h) h d \theta d s .
\end{aligned}
$$


Note that

$$
\|s+\theta t h\| \leq\|s\|+t \leq \sqrt{n}\|s\|_{x}+t \leq \sqrt{n} \epsilon+t .
$$

Hence, by letting $t<\epsilon$, we have

$$
\nabla F(x+s+t h) \in \partial_{(\sqrt{n}+1) \epsilon} F(x),
$$

and consequently,

$$
\nabla F(x+s+t h) h \in \partial_{(\sqrt{n}+1) \epsilon} F(x) h,
$$

which implies (26).

Lemma 3.4. Let $\nabla F_{\epsilon}(x)$ be given by (25). Suppose that $\partial F\left(x^{*}\right)$ is nonsingular. Then, there exist a constant $C_{2}>0$ and $\delta>0$ such that $\nabla F_{\epsilon}(x)$ is nonsingular and

$$
\left\|\nabla F_{\epsilon}(x)^{-1}\right\| \leq C_{2}, \quad \forall x \in S\left(x^{*}, \delta\right), \epsilon>\delta .
$$

Proof. By Proposition 3.1 (d), for every $\mu>0$, there exists $\delta>0$ such that

$$
\partial_{(\sqrt{n}+1) \epsilon} F(x) \subset \partial F\left(x^{*}\right)+\mu B, \quad \forall x \in S\left(x^{*}, \delta\right), \epsilon<\delta .
$$

Since $\partial F\left(x^{*}\right)$ is nonsingular, for $\mu$ sufficiently small, every matrix of $\partial F\left(x^{*}\right)+\mu \bar{B}$ is nonsingular. Now, let

$$
\mathscr{A} \equiv \partial F\left(x^{*}\right)+\mu \bar{B}
$$

Then,

$$
\sigma \equiv \min _{A \in \mathscr{Q}} \min _{\|h\|=1}\|A h\|>0 .
$$

On the other hand, it follows from Lemma 3.3 that

$$
\nabla F_{\epsilon}(x) h \in \partial_{(\sqrt{n}+1) \epsilon} F(x) h, \quad \forall h \in R^{n} .
$$

Hence,

$$
\nabla F_{\epsilon}(x) h \in \mathscr{A} h,
$$

and

$$
\min _{\|h\|=1}\left\|\nabla F_{\epsilon}(x) h\right\| \geq \min _{A \in, \alpha} \min _{\|h\|=1}\|A h\|=\sigma>0,
$$


which implies that $\nabla F_{\epsilon}(x)$ is nonsingular. Letting $C_{2}=1 / \sigma$, we have

$$
\begin{array}{r}
\left\|\nabla F_{\epsilon}(x)^{-1}\right\|=1 / \min _{\|h\|=1}\left\|\nabla F_{\epsilon}(x) h\right\| \leq 1 / \sigma=C_{2}, \\
\forall x \in S\left(x^{*}, \delta\right), \epsilon<\delta,
\end{array}
$$

which completes the proof.

Theorem 3.3. Let $\nabla F_{\epsilon}(x)$ be given by (25). Then, Theorem 3.2 holds for $U_{k}=\nabla F_{E\left(\| x-x^{*} \mid\right)}\left(x_{k}\right)$ in (23).

Proof. Since $\partial F\left(x^{*}\right)$ is nonsingular, it follows from Lemma 3.4 that there exists $\delta>0$ such that $\nabla F_{\epsilon}(x)$ is nonsingular and $\left\|\nabla F_{\epsilon}(x)^{-1}\right\|$ is bounded for all $x \in S\left(x^{*}, \delta\right), \epsilon<\delta$. On other hand, for all $h \in R^{n}$ with $\|h\|=1$, it follows from Lemma 3.3 that

$$
\nabla F_{\epsilon}(x) h \in \partial_{(\sqrt{n}+1) \epsilon} F(x) h, \quad \forall h \in R^{n} .
$$

Since $\partial_{(\sqrt{n}+1) \epsilon} F(x)$ is closed, for every $h$ there exists $U_{h} \in \partial_{(\sqrt{n}+1) \epsilon} F(x)$ such that $\nabla F_{\epsilon}(x) h=U_{h} h$. By Lemma 3.2 and Remark 3.2, $U_{h}$ satisifes (22) for $\epsilon=E\left(\left\|x-x^{*}\right\|\right)$. Therefore, by analogy with the proof of Theorem 3.2, we can easily get the conclusion.

\section{Norm Reducing Technique}

In this section, we discuss briefly the techniques which are likely to be combined with local convergence methods when a starting point is far from a solution point of (1). It is well known that solving the nonlinear simultaneous equations (1) is equivalent to finding the global minimizer of

$$
f(x)=(1 / 2) F(x)^{T} F(x) .
$$

Suppose that $F(x)$ is locally Lipschitz continuous. Then, $f(x)$ is also locally Lipschitz continuous, and

$$
\partial f(x)=\partial F(x)^{T} F(x)
$$

A proof of (27) was given by Clarke; see Theorem 2.6.6, Ref. 14.

General methods for minimizing $f(x)$ can be summarized as follows:

$$
\begin{aligned}
& x_{+}=x_{c}+\alpha d_{c}, \\
& d_{c}=-\arg \min _{v \in \partial f\left(x_{c}\right)}\|v\|,
\end{aligned}
$$

where $x_{c}$ and $x_{+}$denote the current and next approximate root of (1), $\alpha$ is the stepsize, and $d_{c}$ is the search direction. 
Substituting (27) into (28), we have

$$
d_{c}=-\arg \min _{V \in \hat{i} F\left(x_{i}\right)}\left\|V F\left(x_{c}\right)\right\| .
$$

By the Caratheodory theorem, there exist an integer $m$ and $V_{i} \in \partial F\left(x_{c}\right), i \in \bar{m}$, such that every $V_{c} \in \partial F\left(x_{c}\right)$ can be expressed as

$$
V_{c}=\sum_{i \in \bar{m}} \alpha_{i} V_{i},
$$

with

$$
\sum_{i \in \bar{m}} \alpha_{i}=1, \quad \alpha_{i} \geq 0, \quad V_{i} \in \partial F\left(x_{c}\right) .
$$

As a consequence, (29) is equivalent to

$$
\begin{array}{ll}
\min & \left\|\sum_{i \in \bar{m}} \alpha_{i} V_{i}^{T} F\left(x_{c}\right)\right\|, \\
\text { s.t. } & \sum_{i \in \bar{m}} \alpha_{i}=1, \quad \alpha_{i} \geq 0, \\
& V_{i} \in \partial F\left(x_{c}\right), \quad i \in \bar{m},
\end{array}
$$

and

$$
d_{c}=-\sum_{i \in \bar{m}} \bar{\alpha}_{i} V_{i}^{T} F\left(x_{c}\right),
$$

where $\left(\bar{\alpha}_{1}, \ldots, \bar{\alpha}_{m}\right)$ is the solution of $(30)$.

Therefore, if we already know $V_{i} \in \partial F\left(x_{c}\right)$, the search direction may be computed through (30). However, in practice, it is unrealistic to compute all $V_{i} \in \partial F\left(x_{c}\right)$. One possible substitute is to use the former iteration matrices computed at the previous steps. Let

$$
V_{i} \in \partial F\left(x_{i}\right) \text { and } I=\left[i:\left\|x_{c}-x_{i}\right\| \leq \epsilon\right] .
$$

Then, we may compute an approximate search direction by solving

$$
\begin{array}{ll}
\min & \left\|\sum_{i \in I} \alpha_{i} V_{i}^{T} F\left(x_{c}\right)\right\|, \\
\text { s.t. } & \sum_{i \in I} \alpha_{i}=1, \quad \alpha_{i} \geq 0, \\
& V_{i} \in \partial F\left(x_{i}\right), \quad i \in I,
\end{array}
$$

instead of (30). This method is called the bundle-like method. 
An alternative suggested by Fletcher is to use the $L_{1}$-norm, i.e., to convert (1) into the following global minimization problem:

$$
\min \phi(x)=\sum_{i \in \bar{n}}\left|f_{i}(x)\right|
$$

Suppose that $f_{i}(x), i \in \bar{n}$, are regular (Ref. 14). Then, for every $h \in R^{n}$,

$$
\phi^{\prime}(x, h)=\sum_{i \in \alpha(x)} f_{i}^{\prime}(x, h)-\sum_{i \in \beta(x)} f_{i}^{\prime}(x, h)+\sum_{i \in \gamma(x)}\left|f_{i}^{\prime}(x, h)\right|,
$$

where

$$
\begin{aligned}
& \alpha(x)=\left[i \in \bar{n}: f_{i}(x)>0\right], \\
& \beta(x)=\left[i \in \bar{n}: f_{i}(x)<0\right], \\
& \gamma(x)=\left[i \in \bar{n}: f_{i}(x)=0\right] .
\end{aligned}
$$

We may use a trust region method to find a descent direction by solving

$$
\begin{array}{ll}
\min & {\left[\phi(x)+\phi^{\prime}(x, h)+(1 / 2) h^{r} B h\right],} \\
\text { s.t. } & \|h\| \leq \Delta .
\end{array}
$$

We omit further discussion, since it is not the main topic in this paper.

\section{Numerical Experiments}

We implemented the finite-difference approximation method, based on (4) and (18), and the $\epsilon$-generalized Jacobian methods based on (23) for some typical examples. The computations were performed in Matlab. The results show that our methods are efficient.

Example 5.1. Consider the following nonsmooth equations:

$$
F(x)=\left[\begin{array}{l}
\left|x_{1}\right|+\left(x_{2}-1\right)^{2}-1 \\
\left(x_{1}-1\right)^{2}+\left|x_{2}\right|-1
\end{array}\right]=0 .
$$

This problem has two solutions,

$$
x^{*}=(1,1)^{T}, \quad x^{* *}=(0,0)^{T} .
$$


Table 1. Results for Example 5.1.

\begin{tabular}{llcll}
\hline Initial point & $s$ & NIT & Solution & $\|F(\hat{x})\|_{2}$ \\
\hline$(1,0)^{T}$ & 1.E-1 & 11 & $(1,1)^{T}$ & $6.5087 \mathrm{e}-07$ \\
$(1,-1)^{T}$ & $1 . \mathrm{E}-5$ & 6 & $(0,0)^{T}$ & $7.6141 \mathrm{e}-08$ \\
$(-1,0)^{T}$ & $1 . \mathrm{E}-5$ & 4 & $(0,0)^{T}$ & $1.8981 \mathrm{e}-08$ \\
$(-1,-1)^{T}$ & $1 . \mathrm{E}-5$ & 4 & $(0,0)^{T}$ & $2.3110 \mathrm{e}-09$ \\
\hline
\end{tabular}

$F$ is differentiable at $x^{*}$ but nondifferentiable at $x^{* *}$, and it satisfies the conditions of Lemma 3.1. We used the finite-difference approximation method to solve this problem. The numerical results are shown in Table 1, in which $s$ is the parameter in (18), NIT denotes the number of iterations needed to reach the specified precision, "solution" is the exact solution of the equations, to which the iteration sequence converges, and $F(\hat{x})$ is the function evaluated at the computed solution $\hat{x}$.

Example 5.2. Consider the function $F: R^{n} \rightarrow R^{n}$,

$$
F_{i}(x)= \begin{cases}c_{1} g_{1}(x), & \text { if } g_{\imath}(x) \geq 0, \\ c_{2} g_{i}(x), & \text { if } g_{1}(x) \leq 0,\end{cases}
$$

where

$$
g_{i}(x)=i-\sum_{j=1}^{i}\left[\cos \left(x_{j}-1\right)+j\left(1-\cos \left(x_{j}-1\right)\right)-\sin \left(x_{j}-1\right)\right] .
$$

When $c_{1}=c_{2}, F(x)$ is continuously differentiable. Therefore $\left|c_{1}-c_{2}\right|$ may be interpreted as the degree of nondifferentiability of $F$. As it is easily verified, $F(x)$ has the solutions $\left(1+2 k_{1} \pi, \ldots, 1+2 k_{n} \pi\right)^{T}$, where $k_{1}, \ldots, k_{n}$ are arbitrary integers. The example was considered by Martinez and Qi in Ref. 21.

We used the $\epsilon$-generalized Jacobian method to compute this example. An obvious difficulty is how to compute the elements of the $\epsilon$-generalized Jacobian. Let $x$ be the current point at which $F$ is nondifferentiable. In the neighborhood of $x$, find a point $y$ at which $F$ is differentiable and take $U_{k}=\nabla F(y)$. However, it is difficult to design a deterministic algorithm for identifying $y$. In our implementation, we get $y$ by giving a random small perturbation to $x$. If $F$ is still nondifferentiable at $y$, then repeat the above process until finally $F$ is differentiable at $y$. By the Rademacher theorem, $F$ is differentiable almost everywhere. Therefore, the probability that $y$ lies in $D_{F}$ is one. Clearly, running the same program for the same problem at differnt times results in a different numbers of iternations due to different perturbations. In the test, we ran our program 100 times for each case. The 
Table 2. Results for Example 5.2.

\begin{tabular}{rrrr}
\hline$c_{1}$ & $c_{2}$ & ANIT & ANP \\
\hline 1 & -1 & 8.48 & 4.55 \\
20 & -20 & 9.71 & 5.76 \\
100 & -100 & 10.60 & 6.63 \\
\hline
\end{tabular}

termination criterion is

$$
\left\|F\left(x_{k}\right)\right\|_{2} \leq 1.0 \mathrm{E}-6 .
$$

Numerical results are displayed in Table 2 for $n=20$ with initial point $x_{0}=$ $(0, \ldots, 0)^{T}$. In Table 2 , ANIT denotes the average number of iterations needed to reach the specified precision, ANP denotes the average number of perturbations during the whole iterations.

Example 5.3. Consider the following nonlinear complementarity problem: find $x \in R^{4}$ such that

$$
x \geq 0, \quad f(x) \geq 0, \quad x^{T} f(x)=0,
$$

where $f: R^{4} \rightarrow R^{4}$ is given by

$$
\begin{aligned}
& f_{1}(x)=3 x_{1}^{2}+2 x_{1} x_{2}+2 x_{2}^{2}+x_{3}+3 x_{4}-6 \\
& f_{2}(x)=2 x_{1}^{2}+x_{1}+x_{2}^{2}+10 x_{3}+2 x_{4}-2 \\
& f_{3}(x)=3 x_{1}^{2}+x_{1} x_{2}+2 x_{2}^{2}+2 x_{3}+9 x_{4}-9 \\
& f_{4}(x)=x_{1}^{2}+3 x_{2}^{2}+2 x_{3}+3 x_{4}-3
\end{aligned}
$$

The problem can be transformed into the solution of nonsmooth equations:

$$
F(x)=\min \{f(x), x\}=0,
$$

where min refers to the componentwise minimum. The problem has two solutions,

$$
x^{*}=(1,0,3,0)^{T}, \quad x^{* *}=(\sqrt{6} / 2,0,0,0.5)^{T} .
$$

Table 3. Results for Example 5.3.

\begin{tabular}{llll}
\hline Initial point & ANIT & ANP & Solution \\
\hline$(1,0,0,0)^{T}$ & 3 & 0 & $(\sqrt{6} / 2,0,0,0.5)^{T}$ \\
$(1,0,1,-5)^{T}$ & 3 & 0 & $(\sqrt{6} / 2,0,0,0.5)^{T}$ \\
$(1,0,1,0)^{r}$ & 1.96 & 1 & $(1,0,3,0)^{T}$ \\
$(1,0,0,1)^{T}$ & 3.52 & 1 & $(\sqrt{6} / 2,0,0,0.5)^{T}$ \\
$(0,0,0,1)^{T}$ & failed & - & $(\sqrt{6} / 2,0,0,0.5)^{T}$ \\
\hline
\end{tabular}


$F(x)$ is differentiable at $x^{*}$ but nondifferentiable at $x^{* *}$. We used the $\epsilon-$ generalized Jacobian method to solve the problem. Some technique for the implementation of the method has been presented in Example 5.2. Also, we ran the same program 100 times for each case as we did in Example 5.1. The termination criterion is still

$$
\left\|F\left(x_{k}\right)\right\|_{2} \leq 1.0 \mathrm{E}-6 .
$$

\section{References}

1. Robinson, S. M., Local Structure of Feasible Sets in Nonlinear Programming Part 3: Stability and Sensitivity, Mathematical Programming Study, Vol. 30, pp. 45-66, 1987.

2. Robinson, S. M., Newton's Method for a Class of Nonsmooth Functions, Working Paper, Industrial Engineering Department, University of Wisconsin, Madison, Wisconsin, 1988.

3. RoBinson, S. M., An Implicit Function Theorem for B-Differentiable Functions, Working Paper, Industrial Engineering Department, University of Wisconsin, Madison, Wisconsin, 1988.

4. HaRker, P. T., and XIAO, B., Newton's Method for Nonlinear Complementarity Problem: A B-Differentiable Equation Approach, Mathematical Programming, Vol. 48, pp. 339-357, 1990.

5. Pang, J. S., Newton's Method for B-Differentiable Equations, Mathematics of Operation Research, Vol. 15, pp. 311-341, 1990.

6. PANG, J. S., A B-Differentiable Equation-Based, Globally and Locally Quadratically Convergent Algorithm for Nonlinear Programs, Complementarity, and Variational Inequality Problems, Mathematical Programming, Vol. 51, pp. 101$131,1991$.

7. QI, L., Superlinearly Convergent Approximate Newton Methods for LC'-Optimization Problems, Mathematical Programming, Vol. 64, pp. 277-294, 1994.

8. Sun, J., and Ql, L., An Interior Point Algorithm of $O(\sqrt{m}|\log \epsilon|)$ Iterations for $C^{\prime}$-Convex Programming, Mathematical Programming, Vol. 57, pp. 239-257, 1992.

9. XIAO, B., and Harker, P. T., A Nonsmooth Newton Method for Variational Inequalities, Part l: Theory, Mathematical Programming, Vol. 65, pp. 151-194, 1994.

10. XIAo, B., and Harker, P. T., A Nonsmooth Newton Method for Variational Inequalities, Part 2: Numerical Results, Mathematical Programming, Vol. 65, pp. $195-216,1994$.

11. Kojima, M., and Shindo, S., Extensions of Newton and Quasi-Newton Methods to Systems of $P C^{\prime}$-Equations, Journal of the Operation Research Society of Japan, Vol. 29, pp. 352-374, 1986. 
12. IP, C. M., and KyPaRisis, J., Local Convergence of Quasi-Newton Methods for B-Differentiable Equations, Mathematical Programming, Vol. 58, pp. 71-89, 1992.

13. QI, L., and Sun, J., A Nonsmooth Version of Newton's Method, Mathematical Programming, Vol. 58, pp. 353-367, 1993.

14. Clarke, F. K., Optimization and Nonsmooth Analysis, John Wiley and Sons, New York, New York, 1983.

15. PANG, J. S., and QI, L., Nonsmooth Equations: Motivation and Algorithms, SIAM Journal on Optimization, Vol. 3, pp. 443-465, 1993.

16. QI, L., Convergence Analysis of Some Algorithms for Solving Nonsmooth Equations, Mathematics of Operation Research, Vol. 18, pp. 227-244, 1993.

17. Chen, X., and QI, L., A Parameterized Newton Method and a Quasi-Newton Method for Solving Nonsmooth Equations, Computational Optimization and Applications, Vol. 3, pp. 157-179, 1994.

18. Xu, H., A New Version of Newton's Method for Nonsmooth Equations, Numerical Report 158, Dundee University, Dundee, Scotland, 1995.

19. Ortega, J. M., and Rheinboldt, W. C., Iterative Solution of Nonlinear Equations in Several Variables, Academic Press, New York, New York, 1970.

20. Mayne, D. Q., and Polak, E., Nondifferentiable Optimization Adaptive Smoothing, Journal of Optimization Theory and Applications, Vol. 43, pp. 601-613, 1984.

21. Martinez, J. M., and QI, L., Inexact Newton Methods for Solving Nonsmooth Equations, Journal of Computational and Applied Mathematics Vol. 60, pp. $127-145,1995$. 